\title{
Endothelial microvesicles induce blood-brain barrier and neuronal damage caused by intermittent hypoxia through Fas/FasL-caspase8 signaling pathway
}

Qiang Zhang ( $\nabla$ zhangqiangyulv@163.com )

Tianjin Medical University General Hospital, Tianjin Geriatrics Institute

\section{Ying Guo}

Tianjin Medical University General Hospital, Tianjin Geriatrics Institute

Jin Tan

Tianjin Medical University General Hospital, Tianjin Geriatrics Institute Yuyang Miao

Tianjin Medical University General Hospital, Tianjin Geriatrics Institute

\section{Research Article}

Keywords: intermitent hypoxia, microvesicles, oxidative stress

Posted Date: January 27th, 2022

DOI: https://doi.org/10.21203/rs.3.rs-1240723/v1

License: (1) (1) This work is licensed under a Creative Commons Attribution 4.0 International License.

Read Full License 


\section{Abstract}

Background: Obstructive sleep apnea (OSA) is a highly popular disease. Intermittent hypoxia $(\mathrm{IH})$, as the main disease physiological characteristic of OSA, is closely related to the cognitive impairment. Microvesicles (MVs), as intercellular information transfer substances, are closely related to multiple pathological and physiological activities. Endothelial microvesicles (EMVs), as one of the most widely studied microvesicles, has been shown to be associated with the severity of cognitive impairment. However, the effect of $\mathrm{IH}$-induced EMVs on neurocytes is not clear. In our study, we established an IH model in vivo and in vitro to induce EMVs release and to observe its effect on neurocytes function.

Methods: we established animal and cell models of $\mathrm{IH}$, and detected the size and number of EMVs by flow cytometry and nanoparticle tracking analysis. Apoptosis and tight junction proteins were detected by immunoblotting and immunofluorescence. The level of miR126 was detected by PCR.

Results: IH injury model was established to induce B3 to release IH-EMVs. The IH-EMVs from B3 were isolated from the conditioned culture medium and characterized. IH is a potent stimulus for EMV formation and that EMV formed under intermitent hypoxia are compositionally and functionally distinct from those formed under normoxia conditions. Then brain microvascular endothelial cells and neurocytes were then incubated with $\mathrm{N}-\mathrm{EMV}$ s and IH-EMVs respectively for $8 \mathrm{~h}$. We found that B3 cells treated by $\mathrm{IH}-\mathrm{EMV}$ s exhibited reduced tight junction proteins and increased cell apoptosis. In addition, $\mathrm{IH}$ EMVs contained significantly higher level of ROS than EMVs generated from untreated B3, which might be a direct source to trigger a cascade of which might be a direct source to trigger a cascade of myocardial damage.

Conclusion: We showed that EMVs released during $\mathrm{IH}$ injury are pro-apoptotic, pro-oxidative and directly pathogenic to endothelial cells in vitro. EMVs carry ROS and they may impair endotheliocytes by promoting apoptosis and oxidative stress. These findings provide new insights into the pathogenesis of $\mathrm{IH}$.

\section{Background}

OSA, a very common sleep and breathing disorder, is the repetitive collapse of the upper airway during sleep. Intermittent hypoxia $(\mathrm{IH})$ is characterized by repeated hypoxia and reoxygenation, and is considered as the characteristic and the most important pathological and physiological pathways of OSA. Multiple epidemiological studies have already shown an association between obstructive sleep apnea (OSA) and cognitive dysfunction. Compared with those without OSA, patients with OSA had a higher rate of mild cognitive impairment or dementia. Although, OSA has an close relationship with cognitive dysfunction, the underlying pathogenesis of cognitive dysfunction in OSA patients remain less well understood. Upregulation of oxidative stress in OSA plays an important pathogenic role in the milieu of hypoxiainduced cerebral and cardiovascular dysfunctions. Strong evidence underscores that cerebral amyloidogenesis and tau phosphorylation-two cardinal features of Alzheimer's disease (AD), are 
triggered by hypoxia. Mice subjected to hypoxic conditions unambiguously demonstrated upregulation in cerebral amyloid plaque formation and tau phosphorylation, as well as memory deficit. Hypoxia triggers neuronal degeneration and axonal dysfunction in both cortex and brainstem. Consequently, neurocognitive impairment in apneic/hypoxic patients is attributable to a complex interplay between $\mathrm{ClH}$ and stimulation of several pathological trajectories.

Microvesicles (MVs) are small membranous vesicles shed from the plasma membrane directly by budding, size in range from 0.1 to $1 \mu \mathrm{m}$ in diameter. Normal cells can release a small amount of MVs, but MVs are mainly derived from activated or apoptotic cells. Studies have shown that circulating MVs are actively involved in the pathogenesis of $A D$ and cerebrovascular diseases, which share common vascular risk factors. Platelet derived microvesicles are closely related to vascular dementia because of their thrombotic effects, while ha can participate in the transport of $A \beta$ proteins, thus affecting the occurrence of $A D$. Other studies have shown that microvesicles produced by microglia can participate in the transport of $A \beta$ proteins and participate in nerve cell injury, which is closely related to cognitive impairment.

It is reported that endothelial microvesicles are also closely related to cognitive impairment. Endothelial microvesicles (EMVs) increases in cerebrospinal fluid and plasma of AD patients, and can lead to bloodbrain barrier injury, which is closely related to the severity of cognitive impairment. There are also reports that EMV can damage the blood-brain barrier, thereby affecting cognitive function. Studies have shown that endothelial microvesicles are significantly increased in the blood of OSA patients and are closely related to many pathophysiological processes in OSA patients. However, the role of endothelial microvesicles in OSA cognitive impairment has not been reported in detail. In this study, we discussed the effects and mechanisms of endothelial microvesicles on blood-brain barrier and nerve cells under intermittent hypoxia.

\section{Methods}

\section{Culture of endothelial cells and IH exposure}

Brain microvascular endothelial cells (Bend 3, B3) were purchased from the China Infrastructure of Cell Line Resource (Beijing, China), and cultured in DMEM high glucose supplemented with $10 \%$ fetal bovine serum, $1 \%$ penicillin/streptomycin. Cells were maintained at $37^{\circ} \mathrm{C}$ in a humidified incubator with $5 \% \mathrm{CO} 2$.

B3 cells were exposed to $\mathrm{IH}$ until they reached approximately $80 \%$ confluence. The $\mathrm{IH}$ cell model was established according to our previously described protocol. Briefly, the culture medium was changed to fresh serum-free medium before the cells were exposed to $\mathrm{IH}$. B3 cells grown in culture plates were placed in a Plexiglas exposure chamber, which was alternately flushed with a hypoxia gas mixture $\left(1.5 \% \mathrm{O}_{2}, 5 \%\right.$ $\mathrm{CO}_{2}$, and balanced $\mathrm{N}_{2}$, hypoxia phase, $\left.600 \mathrm{~s}\right)$ or normoxia gas mixture $\left(21 \% \mathrm{O}_{2}, 5 \% \mathrm{CO}_{2}\right.$, and balanced $\mathrm{N}_{2}$, reoxygenation phase, $300 \mathrm{~s}$ ). The chamber was equipped with a humidifier, thermostat, and molecular sieve to maintain an inner temperature of $37^{\circ} \mathrm{C}$, humidity of $45 \%$, and relatively germ-free conditions.. 


\section{EMV generation and isolation}

EMV were isolated from the medium of cultured $B 3$ treated with normoxia, intermitent hypoxia $\left(21 \% \mathrm{O}_{2}\right.$, $1.5 \% \mathrm{O}_{2}$ ). Samples were centrifuged at $120 \mathrm{~g}$ for 20 minutes at $20^{\circ} \mathrm{C}$ to obtain cell-free media. MPs were then pelleted from cell-free media by centrifugation at $1500 \times \mathrm{g}$ for 20 minutes at $20^{\circ} \mathrm{C}$. Then samples were centrifuged at $13000 \mathrm{~g}$ for 3 minutes at $20^{\circ} \mathrm{C}$ to obtain endothelial MVs. EMVs were confirmed and quantified by flow cytometry.

\section{Detection of microvesicles by flow cytometry}

To label EMV, resuspension solution containing MVs was incubated with PE-Annexin V (BD Biosciences, USA) for 30 minutes. As a negative control, a subpopulation of MVs was resuspended in Annexin V binding buffer lacking calcium. MVs were centrifuged and resuspended in PBS for cell treatment studies.

\section{Treatment of PC12 cells With Endothelial Microvesicles}

Microvesicles were isolated from cultured B3 in basal conditions or intermitent hypoxia conditions as described above and quantified by flow cytometry (annexin V positivity). PC12 were treated with microparticles $\left(10^{5}\right.$ microvesicles $\left./ \mathrm{mL}\right)$.

\section{Western blot analysis}

PC12 were harvested after co-cultured with N-EMVs or IH-EMVs, B3 were harvested for tight junction protein expression detection. Proteins were extracted from B3 cells using a protein extraction kit (Promega, Madison, $\mathrm{WI}$ ) in accordance with the manufacturer's instructions. Protein concentrations were measured using a bicinchoninic acid assay kit (Thermo Fisher Scientific, Waltham, MA). Protein sample and prestained molecular weight markers (Thermo Fisher Scientific) were applied to $10 \%$ or $12 \%$ sodium dodecyl sulfate-polyacrylamide gel electrophoresis and transferred onto polyvinylidene difluoride membranes (Merck Millipore, Burlington, MA). The membranes were blocked by $5 \%$ non-fat dry milk in Tris-buffered saline and Tween 20 for $2 \mathrm{~h}$ at room temperature, and then incubated with primary antibodies at $4{ }^{\circ} \mathrm{C}$ overnight. The appropriate horseradish peroxidase-conjugated secondary IgG was incubated with the membranes for $1 \mathrm{~h}$ at room temperature. Chemiluminescence was imaged in a Chemi Doc XRS+ WB molecular imager using Image Lab software (Bio-Rad Laboratories, Herculaes, CA), and band intensity was measured by ImageJ software. GAPDH (1:1000, CST, USA ) was used to normalize protein loading. The following primary antibodies were used: Fas (1:1000, Abcam, USA), FasL (1:1000, Abcam, USA) and Caspase-8 (1:1000, Abcam, USA), Bax (1:200, Santa, USA) and Bcl-2(1:200, Santa, USA). 


\section{Immunofluorescence assay for tight junction proteins}

After the experimental conditions were established, the B3 cells were cultured on coverslips, and then cultured with $\mathrm{N}-\mathrm{EMV}$ s and $\mathrm{IH}$-EMVs for $8 \mathrm{~h}$. B3 cells were washed with phosphate-buffered saline and fixed with $4 \%$ paraformaldehyde for $15 \mathrm{~min}$. Then, cells were permeabilized with $0.1 \%$ Triton X-100 for 10 min and blocked with $3 \%$ bovine serum albumin in buffer for $30 \mathrm{~min}$ at room temperature. Subsequently, cells were incubated with primary antibodies(Claudin-5, 1:50;occludin-1, 1:50) over night at $4{ }^{\circ} \mathrm{C}$. The appropriate FITC-labeled secondary antibody was incubated with the slides for $1 \mathrm{~h}$ at room temperature. Apoptosis was assessed with a TdTdUTP nick-end labeling (TUNEL) apoptosis assay kit (Roche, Indianapolis, IN). The slides were incubated with TUNEL reaction mixture for $1 \mathrm{~h}$ at $37^{\circ} \mathrm{C}$. Finally, slides were washed and nuclei were stained with DAPI. Images were obtained using a Fluorescence microscope and Zen software.

\section{Overexpression of miR-126 in B3 cells}

The lentivirus containing murine miR-126 (Lenti-miR126) and lentivirus containing green fluorescence protein (Lenti-GFP) were obtained from GenePharma (GenePharma Co, Ltd, Shanghai, China). B3 cells were seeded in 6-well plates $\left(1 \times 10^{5} /\right.$ well) for a confluence of $70 \%$; then, lentivirus was added (at $5 \times 10^{6}$ infectionforming units) for $6 \mathrm{~h}$, after which the serum-free medium was changed by $10 \%(\mathrm{v} / \mathrm{v})$ FBS medium for $72 \mathrm{~h}$. The level of miR-126 in B3 cells and EMVs were analysed by real-time PCR (RT-PCR).

\section{Gene expression analysis}

The levels of miR-126 in EPCs and EPC-MVs were analysed. Briefly, total RNA was extracted by using miRcute miRNA isolation kit according to the manufacturer's instructions. cDNA was synthesized using miRcute miRNA First-Strand cDNA Synthesis kit. RT-PCR was conducted with miR-126 specific primers and miRcute miRNA qPCR Detection Kit (SYBR Green) (Tiangen, Beijing, China). Small nuclear RNA U6

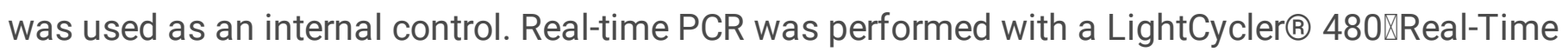
thermal cycler (Roche, Basel, Swiss). The cycling condition was carried out as follows: $95^{\circ} \mathrm{C}$ for $30 \mathrm{~s}$, followed by 40 cycles of $95^{\circ} \mathrm{C}$ for $5 \mathrm{~s}$ and $60^{\circ} \mathrm{C}$ for $20 \mathrm{~s}$. The relative quantification of gene expression was presented with the values of $2-\Delta \Delta C T$ method.

\section{Statistical analysis}

All statistical tests were performed with SPSS 16.0 (SPSS Inc., Chicago, IL). All data are based on at least three independent experiments and are expressed as the mean \pm standard error of the mean (SEM). Results between groups were compared by Student's $t$ test or one-way analysis of variance. A p-value less than 0.05 was considered significant. 


\section{Results}

\section{The characters of EMVs}

We examined EMVs released from IH group using flow cytometry and NTA. As shown in Fig. 1, We examined the effect of intermitent hypoxia on EMV release from cultured B3 using flow cytometry and NTA. The vesicless in the size of $0.1-1 \mu \mathrm{m}$ were screened by flow cytometry (Fig. 1a). The final concentration of MVs is calculated by the number of counting beads(Fig. 1b). NTA analysis showed that EMVs were in size of $100 \mathrm{~nm}$ to $400 \mathrm{~nm}$ (Fig. 1c-d).

\section{IH-EMV reduced the permeability of BBB}

To determine the effect of IH-EMVs might be responsible for B3 cells injury, we treated B3 with N-EMV and IH-EMV, using Western blot analysis to detect the expression of occuludin and claudin5 in B3 cells of every group, it was observed that occuludin and claudin 5 gradually decreased in B3 cells of IH-EMV group (vs. NC; $p<0.01$; Fig. 2a). We also tested the expression of occuludin and claudin 5 production in B3 cells treated with EMVs, and the results were consistent with those of Immunofluorescence assay. Similiarly, immunofluorescence assay showed that IH-EMV could reduced the expression of occuludin and claudin 5 in B3 cells (Fig. 2b-c).

\section{IH-EMVs increased the apoptosis of neurocytes via modulating the expression of Fas/FasL-Caspase 8}

we monitored the cleaved Caspase-8 and Fas/FasL levels, which are associated with induction of apoptosis, by western blot. Results showed that cleaved Caspase-8 protein expression was significantly increased and Fas and FasL protein expression obviously increased by IH-EMVs treatment(vs. NC; $p$ $<0.01$; Fig. 3a).In addition, we monitored the $\mathrm{Bcl}-2$ and Bax levels, which are associated with induction of apoptosis, by western blot. Results showed that $\mathrm{Bcl}-2$ protein expression was significantly decreased whereas Bax protein expression was significantly increased by IH-EMVs treatment (vs. NC; $p<0.01$; Fig. $3 b)$. Then, we used the TUNEL to test cell apoptosis, result showed that the IH-EMVs significantly increased the apoptosis of PC12 cells while N-EMVs did not have such effect (vs. NC; $p<0.01$; Fig. 3c).

To further examine the role of Fas/FasL pathway, we added antagonist KP7-6 to the cell culture medium of IH group. Western blot showed that cleaved Caspase-8 protein expression was significantly increased and Fas and FasL protein expression obviously increased by KP7-6 treatment(vs. NC; $p<0.01$; Fig. 4a).

In addition, we monitored the Bcl-2 and Bax levels, which are associated with induction of apoptosis, by western blot. Results showed that Bcl-2 protein expression was significantly decreased whereas Bax protein expression was significantly increased by KP7-6 treatment (vs. NC; $p<0.01$; Fig. 4b). Then, we used 
the TUNEL to test cell apoptosis, result showed that the KP7-6 significantly increased the apoptosis of PC12 cells while N-EMVs did not have such effect (vs. NC; $p<0.01$; Fig. 4c).

\section{The up-regulation of Fas / FasL expression in neurocytes was related to the ROS IH-EMV carried}

To determine which factor in EMV might be responsible for oxidative stress, we added antioxidants NAC to the cell culture medium of $\mathrm{IH}$ group. We assessed Fas and FasL of all groups. As shown in Fig 5 , Western blot showed that Fas and FasL protein expression obviously decreased by NAC treatment. (vs. IH-EMV; $p<0.05$; Fig. 5a-b).

\section{IH-EMV regulated the ROS-Fas/FasL-Caspase8 pathway through miR126}

RT-PCR showed that IH reduced miR126 level in IH group and lenti-miR126 transfection increased the miR126 level in IH+miR126 group (vs. NC; $p<0.01$; Fig. 6a-c). And the RT-PCR results showed that miR126 in EMVs derived from lenti-miR126 transfection PC12 cells, were increased whereas miR126 in IH-EMVs were decreased (vs. IH-EMV; $p<0.01$; Fig. 6d).

\section{Conclusion}

There are three major findings in this study. Firstly, we demonstrated that N-EMVs released from normal conditions increased cell proliferation, and decreased cell apoptosis, while IH-EMVs obtained from IH condition had opposite effects. Moreover, we revealed that the effects of EMVs were linked with Fas/FasL, Bcl-2 and Caspase-8 signaling pathways. Secondly, N-EMVs and IH-EMVs played different roles in regulating BBB function via modulating the expression of Claudin-5 and Oclludin in ECs. Finally, we demonstrated that IH-EMVs effect the ROS-Fas/FasL-Caspase8 pathway by miR126 (Fig. 7).

MVs are submicron membrane fragments released from virtually all cell types upon activation, apoptosis and stress, which have been documented as potential biomarkers and indicators for diseases. Of note, several studies have demonstrated that MVs released from different stimuli exert different functions to recipient cells. Herein, Pan verified that EMVs fused with astrocytes and regulated the proliferation, apoptosis and GFAP expression of astrocytes. Moreover, Pan found that the $\mathrm{IH}$ - EMVs inhibited the proliferation and the GFAP expression of astrocytes. GFAP has been considered as a specific marker of astrocyte activation. These indicated that N-EMVs may help maintaining astrocyte function and internal environment homeostasis of the brain, while IH-EMVs showed opposite effects on astroctyes, which may participate in the pathological processes of ischemic damage. In fact, activation of astrocytes may play controversial function roles in cerebral damage. For example, astrocyte over-activation might inhibit axonal regeneration by elevating various inhibitory molecules, such as chondroitin sulface proteoglycans. 
Apoptosis can be induced by an extrinsic death receptor pathway or an intrinsic mitochondrial pathway, which causes organ failure in response to various harmful stimuli. Fas is a classical death receptor that belongs to the tumor necrosis factor (TNF) family, whereas Fas ligand (FasL) is a type II membrane protein that binds Fas. The interaction between Fas and FasL can occur on many cell types and triggers apoptosis. Specifically, the interaction between Fas and FasL leads to caspase-8 cleavage activation from procaspase-8, which in turn activates caspase-3. FasL-mediated apoptosis is closely connected to various pathophysiological conditions, such as $\mathrm{I} / \mathrm{R}$ injury and congestive heart failure.

Hypoxia induces apoptosis by increasing Fas expression in various cell types. In addition, hypoxia induces ROS generation, which stimulates the Fas-mediated apoptotic signaling pathway. During oxidative stress, apoptosis is induced by Fas, and caspase subtypes are activated by proteolytical cleavage of critical cellular.proteins, ultimately leading to cell death $(26,36)$. The present study demonstrates that the inhibition of Fas in stem cells significantly downregulates expression of caspases and apoptosis-related proteins (Bax, cytochrome-c) relative to nontreatment. The Fas/FasL complex was found also to be a critical mediator in myocardial injury. Fas-mediated apoptosis is sparked by FasL binding, which initiates the caspase cascade.

Furthermore, oxidative stress might play a role in releasing circulating miR-126-3p as oral treatment with the antioxidant $\mathrm{N}$-acetylcysteine (NAC) was found to prevent the maximal exercise-induced increase of circulating miR-126-3p in patients with intermittent claudication. Wang et al. reported that miR-126-3p contained in endothelial progenitor cell-derived microvesicles (EPC-MVs) can suppress oxidative stress and promote angiogenesis of endothelial cells via the PI3K/eNOS/NO pathway. They also showed that miR-126-3p was increased in MVs released from EPCs cultured in a serum deprived medium (starvation stress), whereas it was decreased in MVs released from EPCs cultured in a serum-deprived medium containing tumor necrosis factor-a (TNFa) (apoptotic stress). This example clearly showed that oxidative stress can be a regulator of extracellular miRNAs, and can be regulated by extracellular miRNAs.

In conclusion, the present study demonstrates that IH-EMVs havs effects on regulating BBB integrity and neurocytes apoptosis via regulating the ROS-Fas/FasL-caspase-8 signal pathways. These data suggest that EMVs may actively promote the progression of cognitive impairment, and that this process may be accelerated in diseases characterized by hypoxic disease.Thus, inhibiting of EMV-based signaling may be a new approach to the treatment of cognitive impairment associated with intermittent hypoxia.

\section{Abbreviations}

OSA:Obstructive sleep apnea; IH:Intermittent hypoxia; MVs: Microvesicles; EMVs:Endothelial microvesicles; AD: Alzheimer's disease

\section{Declarations}

\section{Acknowledgements}




\section{Authors' contributions}

Ying Guo wrote the manuscript. All authors read and approved the final manuscript.

\section{Funding}

This work was supported by the Major Research Plan of National Natural Science Foundation of China (Grant No.92163213) , General Program of National Natural Science Foundation of China (Grant No. 81970085) and the Tianjin science and sechnology plan project (Grant No. 17ZXMFSY00080).

\section{Availability of data and materials}

All data generated or analyzed during this study are included in this published article.

\section{Ethics approval and consent to participate}

Not applicable.

\section{Consent for publication}

Not applicable.

\section{Competing interests}

The authors declare that they have no conflict of interest.

\section{References}

1. Drager LF, Polotsky VY, Lorenzi-Filho G. Obstructive sleep apnea: an emerging risk factor for atherosclerosis. Chest. 2011;140:534-42.

2. Amin Z, Amin HZ, Amin LZ. Obstructive Sleep Apnea and Atherosclerosis. Acta medica Indonesiana. 2016;48:63-7.

3. Ryan S, Taylor CT, McNicholas WT. Selective activation of inflammatory pathways by intermittent hypoxia in obstructive sleep apnea syndrome. Circulation. 2005;112:2660-7. 
4. Badran M, Ayas N, Laher I. Cardiovascular complications of sleep apnea: role of oxidative stress. Oxid Med Cell Longev. 2014;2014:985258.

5. Jelic S, Le Jemtel TH. Inflammation, oxidative stress, and the vascular endothelium in obstructive sleep apnea. Trends in cardiovascular medicine. 2008;18:253-60.

6. Lattimore JD, Celermajer DS, Wilcox I. Obstructive sleep apnea and cardiovascular disease. Journal of the American College of Cardiology. 2003;41:1429-37.

7. Lee TH, D'Asti E, Magnus N, Al-Nedawi K, Meehan B, Rak J. Microvesicles as mediators of intercellular communication in cancer-the emerging science of cellular 'debris'. Seminars in immunopathology. 2011;33:455-67.

8. Yuana Y, Sturk A, Nieuwland R. Extracellular vesicles in physiological and pathological conditions. Blood reviews. 2013;27:31-9.

9. Schiera G, Di Liegro CM, Di Liegro I. Extracellular Membrane Vesicles as Vehicles for Brain Cell-to-Cell Interactions in Physiological as well as Pathological Conditions. Biomed Res Int. 2015;2015:152926.

10. Burger D, Schock S, Thompson CS, Montezano AC, Hakim AM, Touyz RM. Microparticles: biomarkers and beyond. Clinical science. 2013;124:423-41.

11. Burger D, Kwart DG, Montezano AC, Read NC, Kennedy CR, Thompson CS, et al. Microparticles induce cell cycle arrest through redox-sensitive processes in endothelial cells: implications in vascular senescence. Journal of the American Heart Association. 2012;1:e001842.

12. Jy W, Jimenez JJ, Mauro LM, Horstman LL, Cheng P, Ahn ER, et al. Endothelial microparticles induce formation of platelet aggregates via a von Willebrand factor/ristocetin dependent pathway, rendering them resistant to dissociation. Journal of thrombosis and haemostasis : JTH. 2005;3:1301-8.

13. Schock SC, Edrissi H, Burger D, Cadonic R, Hakim A, Thompson C. Microparticles generated during chronic cerebral ischemia deliver proapoptotic signals to cultured endothelial cells. Biochem Biophys Res Commun. 2014;450:912-7.

14. Brodsky SV, Zhang F, Nasjletti A, Goligorsky MS. Endothelium-derived microparticles impair endothelial function in vitro. American journal of physiology Heart and circulatory physiology. 2004;286:H1910-5.

15. Cheng F, Tao J, Feng JQ, Yang CT, Wang Y, Zhang YY, et al. [Role of NADPH oxidase in endothelial cell dysfunction induced by endothelial microparticles]. Nan fang yi ke da xue xue bao $=$ Journal of Southern Medical University. 2010;30:1103-6.

16. Jansen F, Yang X, Franklin BS, Hoelscher M, Schmitz T, Bedorf J, et al. High glucose condition increases NADPH oxidase activity in endothelial microparticles that promote vascular inflammation. Cardiovascular research. 2013;98:94-106.

17. Wang SX, Zhang Q, Shang M, Wei S, Liu M, Wang YL, et al. Microvesicles derived from hypoxia/reoxygenation-treated human umbilical vein endothelial cells impair relaxation of rat thoracic aortic rings. Zhongguo ying yong sheng li xue za zhi = Zhongguo yingyong shenglixue zazhi $=$ Chinese journal of applied physiology. 2014;30:560-6. 
18. He Q, Yang QC, Zhou Q, Zhu H, Niu WY, Feng J, et al. Effects of varying degrees of intermittent hypoxia on proinflammatory cytokines and adipokines in rats and 3T3-L1 adipocytes. PLoS One. 2014;9:e86326.

19. Shah MD, Bergeron AL, Dong JF, Lopez JA. Flow cytometric measurement of microparticles: pitfalls and protocol modifications. Platelets. 2008;19:365-72.

20. Teoh NC, Ajamieh H, Wong HJ, Croft K, Mori T, Allison AC, et al. Microparticles mediate hepatic ischemia-reperfusion injury and are the targets of Diannexin (ASP8597). PLoS One. 2014;9:e104376.

21. Zhang Q, Shang M, Zhang M, Wang Y, Chen Y, Wu Y, et al. Microvesicles derived from hypoxia/reoxygenation-treated human umbilical vein endothelial cells promote apoptosis and oxidative stress in H9c2 cardiomyocytes. BMC cell biology. 2016;17:25.

22. Watts JA, Lee YY, Gellar MA, Fulkerson MB, Hwang SI, Kline JA. Proteomics of microparticles after experimental pulmonary embolism. Thrombosis research. 2012;130:122-8.

23. Berchem G, Noman MZ, Bosseler M, Paggetti J, Baconnais S, Le Cam E, et al. Hypoxic tumor-derived microvesicles negatively regulate NK cell function by a mechanism involving TGF-beta and miR23a transfer. Oncoimmunology. 2016;5:e1062968.

24. Burger D, Turner M, Munkonda MN, Touyz RM. Endothelial Microparticle-Derived Reactive Oxygen Species: Role in Endothelial Signaling and Vascular Function. Oxid Med Cell Longev. 2016;2016:5047954.

25. Burger D, Turner M, Xiao F, Munkonda MN, Akbari S, Burns KD. High glucose increases the formation and pro-oxidative activity of endothelial microparticles. Diabetologia. 2017;60:1791-800.

26. Tuleta I, Franca CN, Wenzel D, Fleischmann B, Nickenig G, Werner N, Skowasch D: Hypoxia-induced endothelial dysfunction in apolipoprotein E-deficient mice; effects of infliximab and L-glutathione. Atherosclerosis 2014, 236(2):400-410.

27. Teoh NC, Ajamieh H, Wong HJ, Croft K, Mori T, Allison AC, Farrell GC: Microparticles mediate hepatic ischemia-reperfusion injury and are the targets of Diannexin (ASP8597). PLoS One 2014, 9(9):e104376.

28. Hu Y, Yan R, Zhang C, Zhou Z, Liu M, Wang C, Zhang H, Dong L, Zhou T, Wu Y et al: High-Mobility Group Box 1 From Hypoxic Trophoblasts Promotes Endothelial Microparticle Production and Thrombophilia in Preeclampsia. Arterioscler Thromb Vasc Biol 2018, 38(6):1381-1391.

29. Zhao XP, Wang M, Song Y, Song K, Yan TL, Wang L, Liu K, Shang ZJ: Membrane microvesicles as mediators for melanoma-fibroblasts communication: roles of the VCAM-1/VLA-4 axis and the ERK1/2 signal pathway. Cancer Lett 2015, 360(2):125-133.

30. Berchem G, Noman MZ, Bosseler M, Paggetti J, Baconnais S, Le Cam E, Nanbakhsh A, Moussay E, Mami-Chouaib F, Janji B et al: Hypoxic tumor-derived microvesicles negatively regulate NK cell function by a mechanism involving TGF-beta and miR23a transfer. Oncoimmunology 2016, 5(4):e1062968.

31. Watts JA, Lee YY, Gellar MA, Fulkerson MB, Hwang SI, Kline JA: Proteomics of microparticles after experimental pulmonary embolism. Thrombosis research 2012, 130(1):122-128. 
32. Falati S, Liu Q, Gross P, Merrill-Skoloff G, Chou J, Vandendries E, Celi A, Croce K, Furie BC, Furie B: Accumulation of tissue factor into developing thrombi in vivo is dependent upon microparticle $\mathrm{P}$ selectin glycoprotein ligand 1 and platelet P-selectin. J Exp Med 2003, 197(11):1585-1598.

33. Jansen F, Yang X, Hoyer FF, Paul K, Heiermann N, Becher MU, Abu Hussein N, Kebschull M, Bedorf J, Franklin BS et al: Endothelial microparticle uptake in target cells is annexin I/phosphatidylserine receptor dependent and prevents apoptosis. Arterioscler Thromb Vasc Biol 2012, 32(8):1925-1935.

34. Wei X, Liu C, Wang H, Wang L, Xiao F, Guo Z, Zhang H: Surface Phosphatidylserine Is Responsible for the Internalization on Microvesicles Derived from Hypoxia-Induced Human Bone Marrow Mesenchymal Stem Cells into Human Endothelial Cells. PLoS One 2016, 11(1):e0147360.

35. Faille D, El-Assaad F, Mitchell AJ, Alessi MC, Chimini G, Fusai T, Grau GE, Combes V: Endocytosis and intracellular processing of platelet microparticles by brain endothelial cells. J Cell Mol Med 2012, 16(8):1731-1738.

\section{Figures}




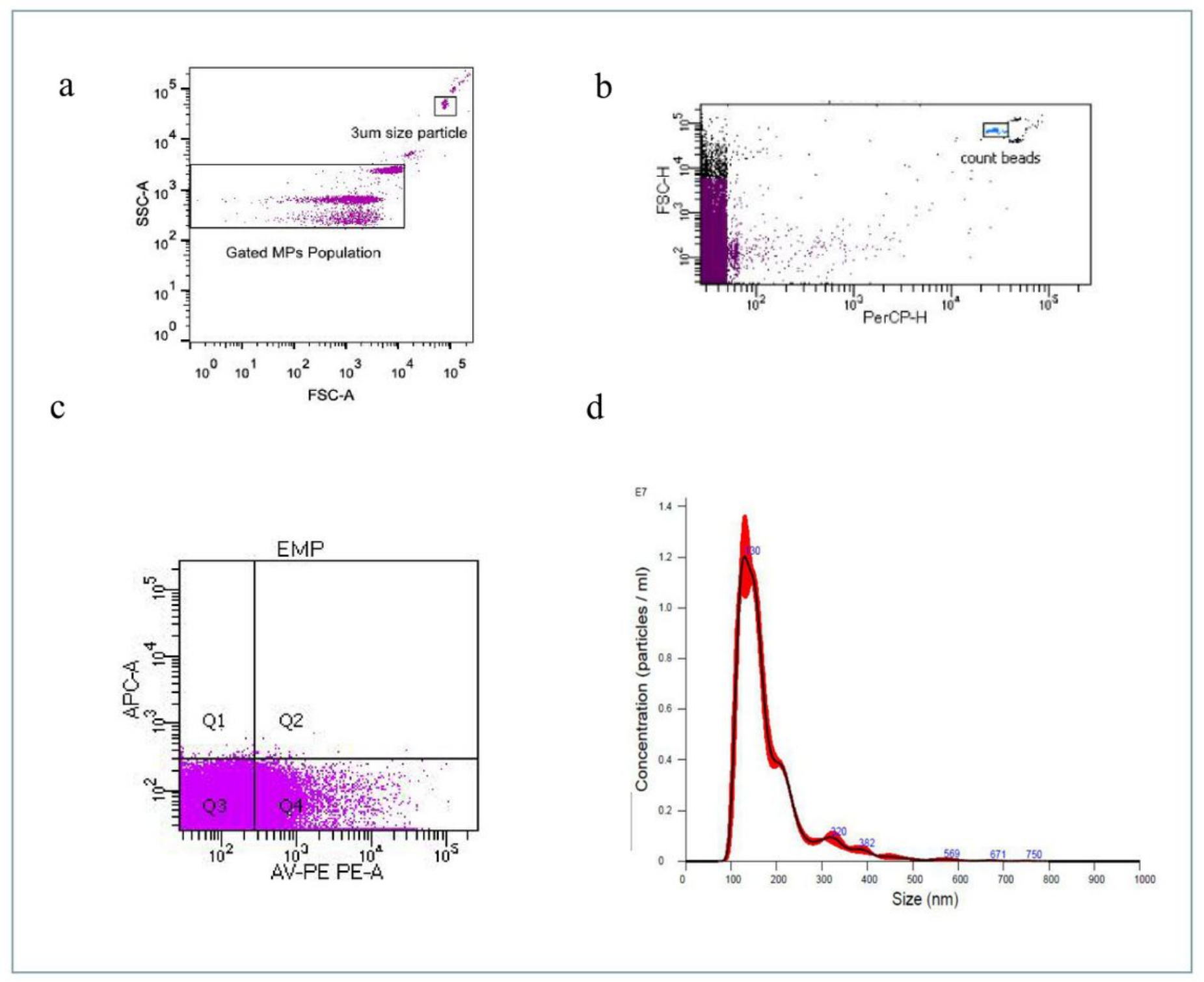

\section{Figure 1}

The characters of EMVs. (a) The vesicles in the size of 0.1-1 $\mu \mathrm{m}$ were screened by flow cytometry. (b) The final concentration of EMVs is calculated by the number of counting beads. EMV levels in cultured B3 cells were assessed by (c) annexin V labelling and flow cytometry or (d) NTA. 

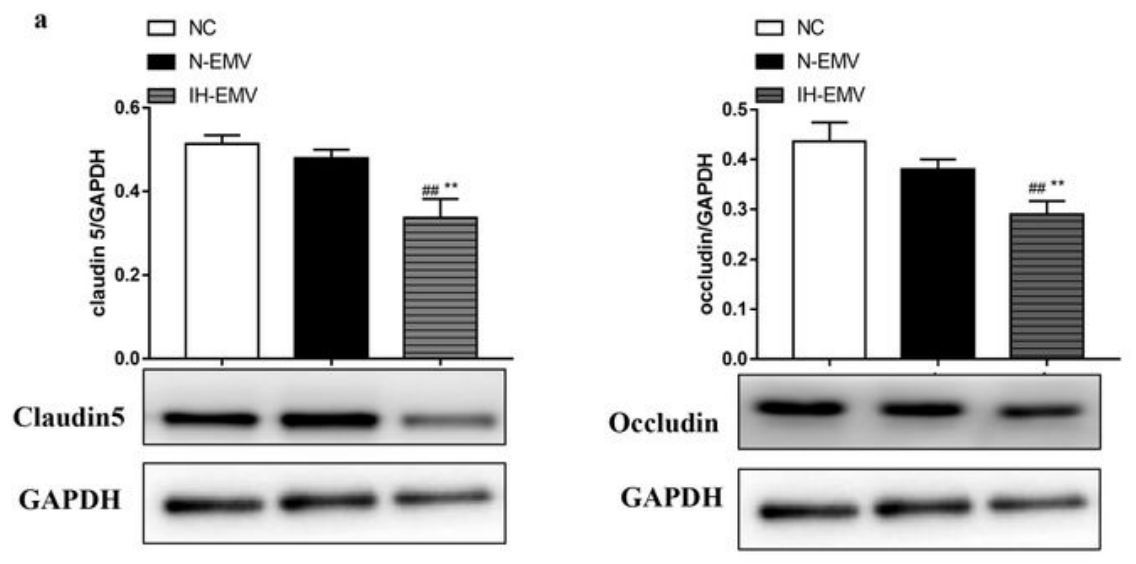

b
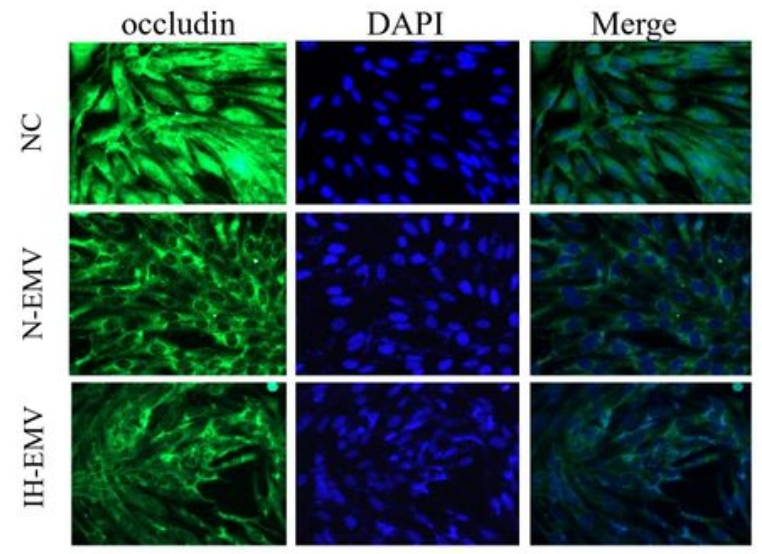

c

DAPI

Merge

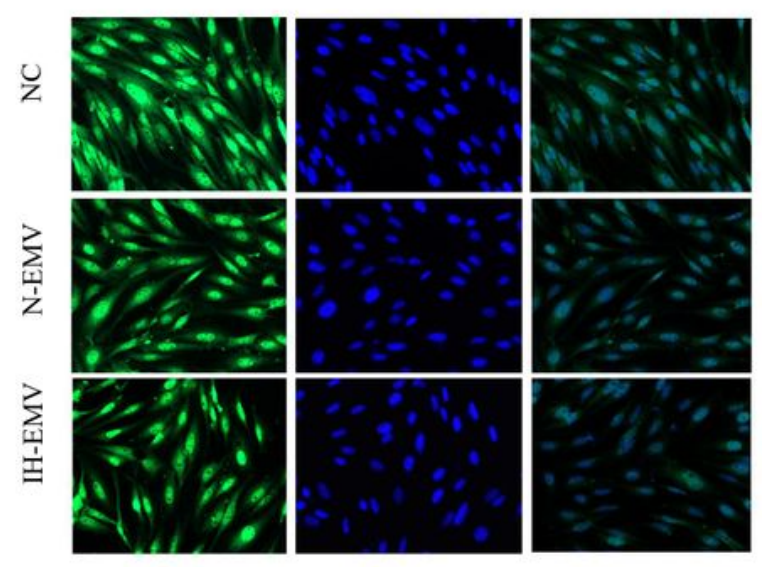

\section{Figure 2}

IH-EMV reduced the permeability of BBB. (a) The expression of occuludin and claudin 5 in B3 cells was assessed by Western blot in NC, N-EMV and IH-EMV group. (b-c)The expression of occuludin and claudin 5 in B3 cells was assessed by Immunofluorescence in NC, N-EMV and IH-EMV group. ${ }^{\# \#} p<0.01$ vs NC, $* \star p p<0.01$ vs N-EMVs. 

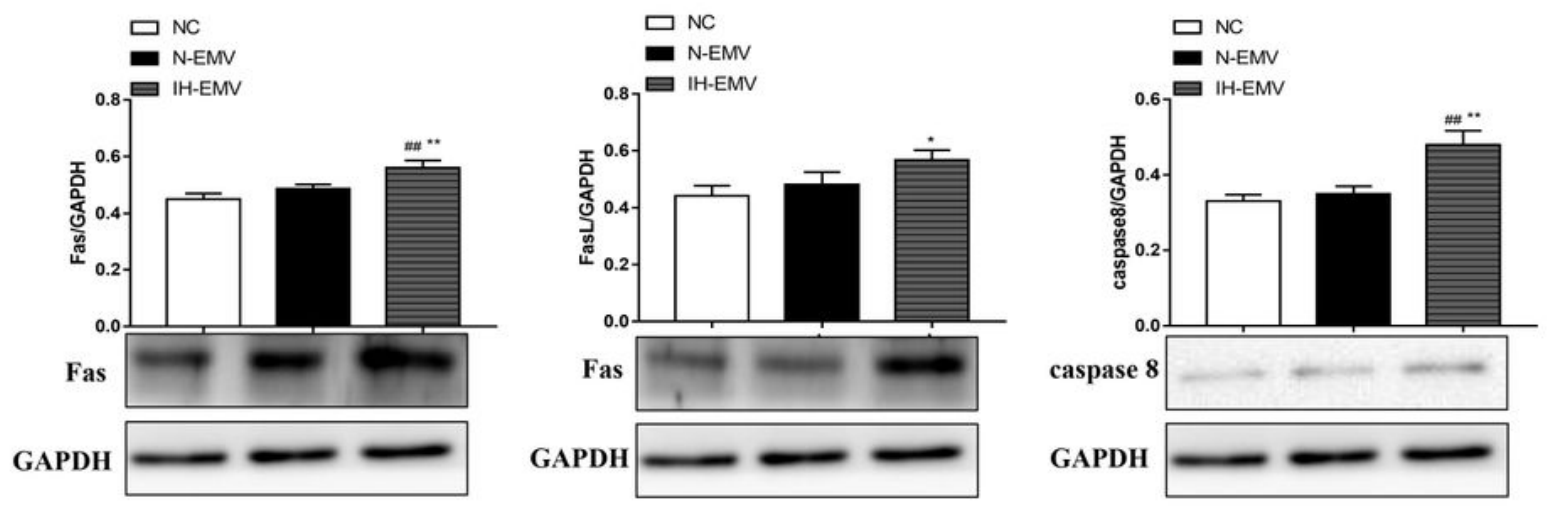

b
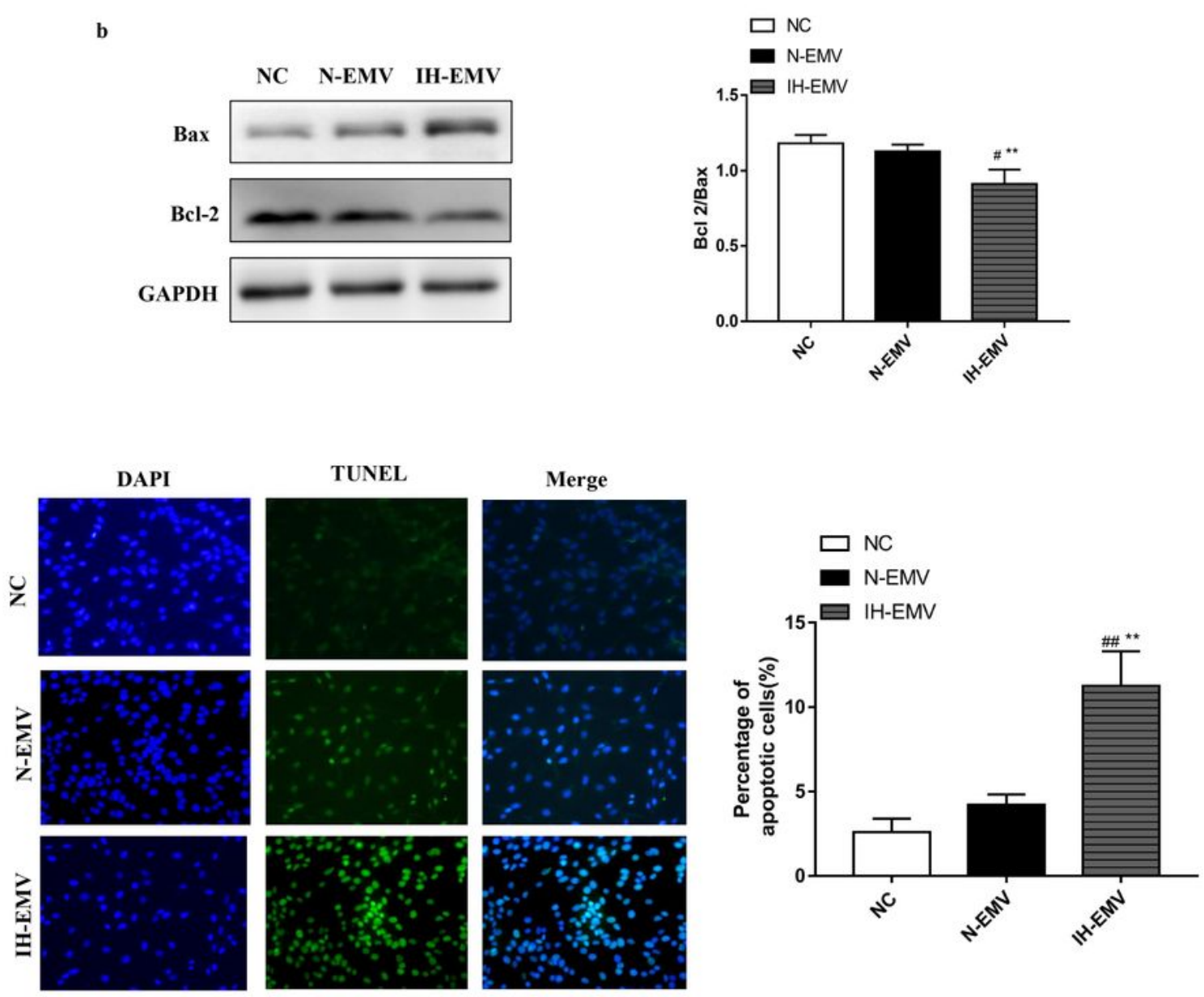

\section{Figure 3}

IH-EMVs increased the apoptosis of neurocytes via modulating the expression of Fas/FasL-Caspase 8.

(a) The expression of Fas, FasL and Caspase 8 in PC12 cells was assessed by Western blot in NC, N-EMV and IH-EMV group. (b) The expression of bax and bcl 2 in PC12 cells was assessed by Western blot in NC, $\mathrm{N}$-EMV and IH-EMV group. (c) The apoptosis of PC12 cells was assessed by TUNEL. ${ }^{\mathrm{p}}<0.05 \mathrm{vs} \mathrm{NC}$, \#\# $<0.01$ vs NC, ${ }^{*} p<0.05$ vs N-EMVs, ** $p<0.01$ vs N-EMVs. 

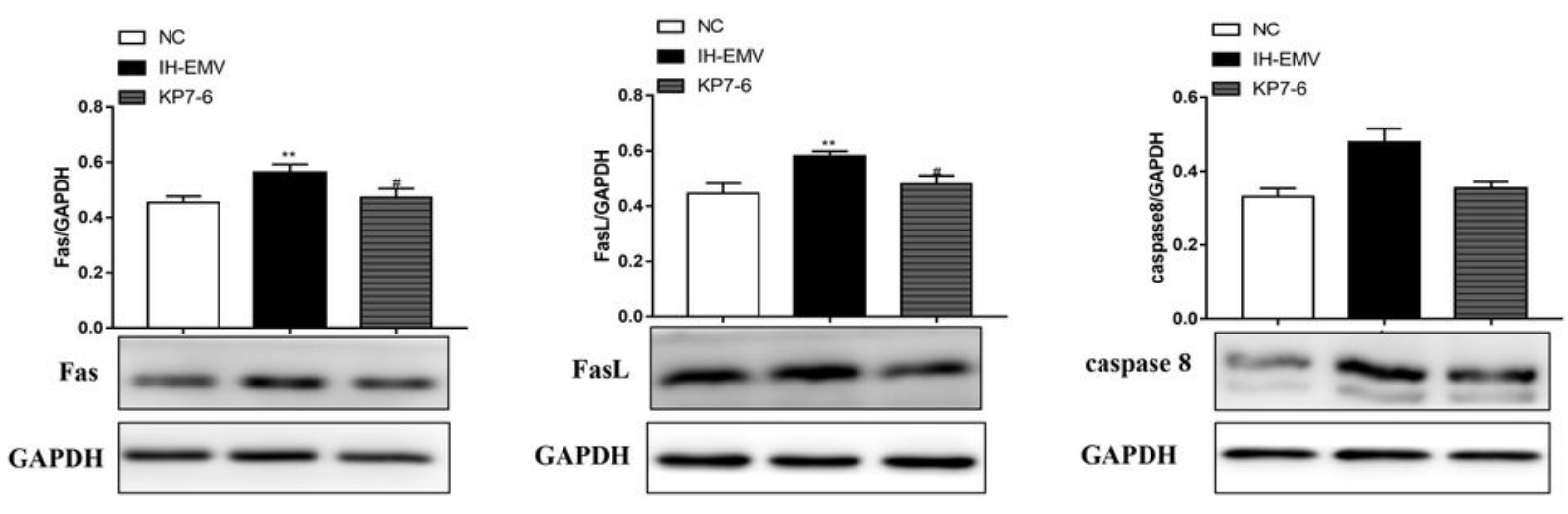

b
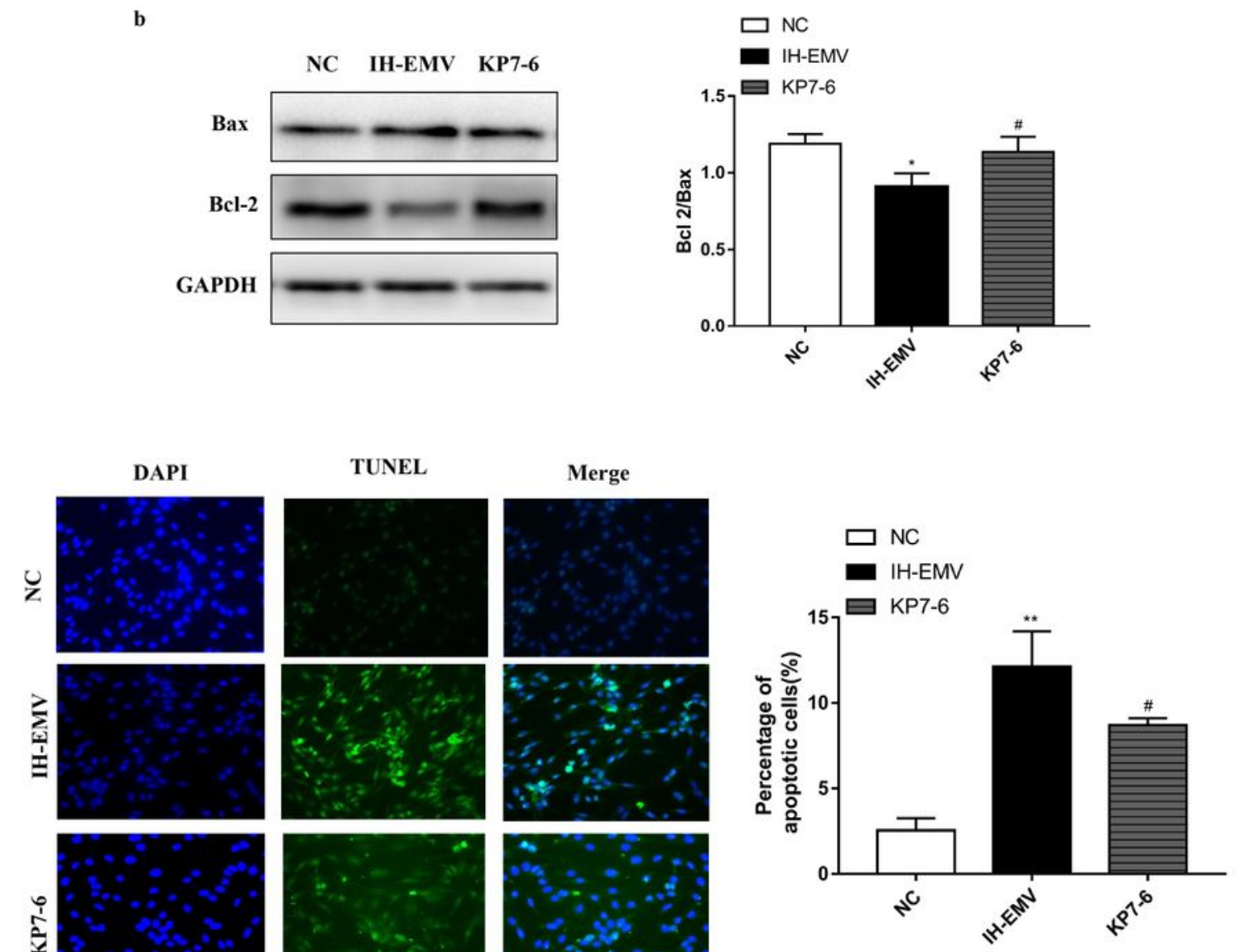

\section{Figure 4}

To further examine the role of Fas/FasL pathway, we added antagonist KP7-6 to the cell culture medium of IH group. (a) The expression of Fas, FasL and Caspase 8 in PC12 cells was assessed by Western blot in NC, IH-EMV and KP7-6 group.(b) The expression of bax and bcl 2 in PC12 cells was assessed by Western blot in NC, IH-EMV and KP7-6. (c) The apoptosis of PC12 cells was assessed by TUNEL in NC, IHEMV and KP7-6 group. ${ }^{*} \mathrm{p}<0.05$ vs NC, ${ }^{\# \#} \mathrm{p}<0.01$ vs NC, ${ }^{*} \mathrm{p}<0.05$ vs N-EMVs, ${ }^{* *} \mathrm{p}<0.01$ vs N-EMVs. 


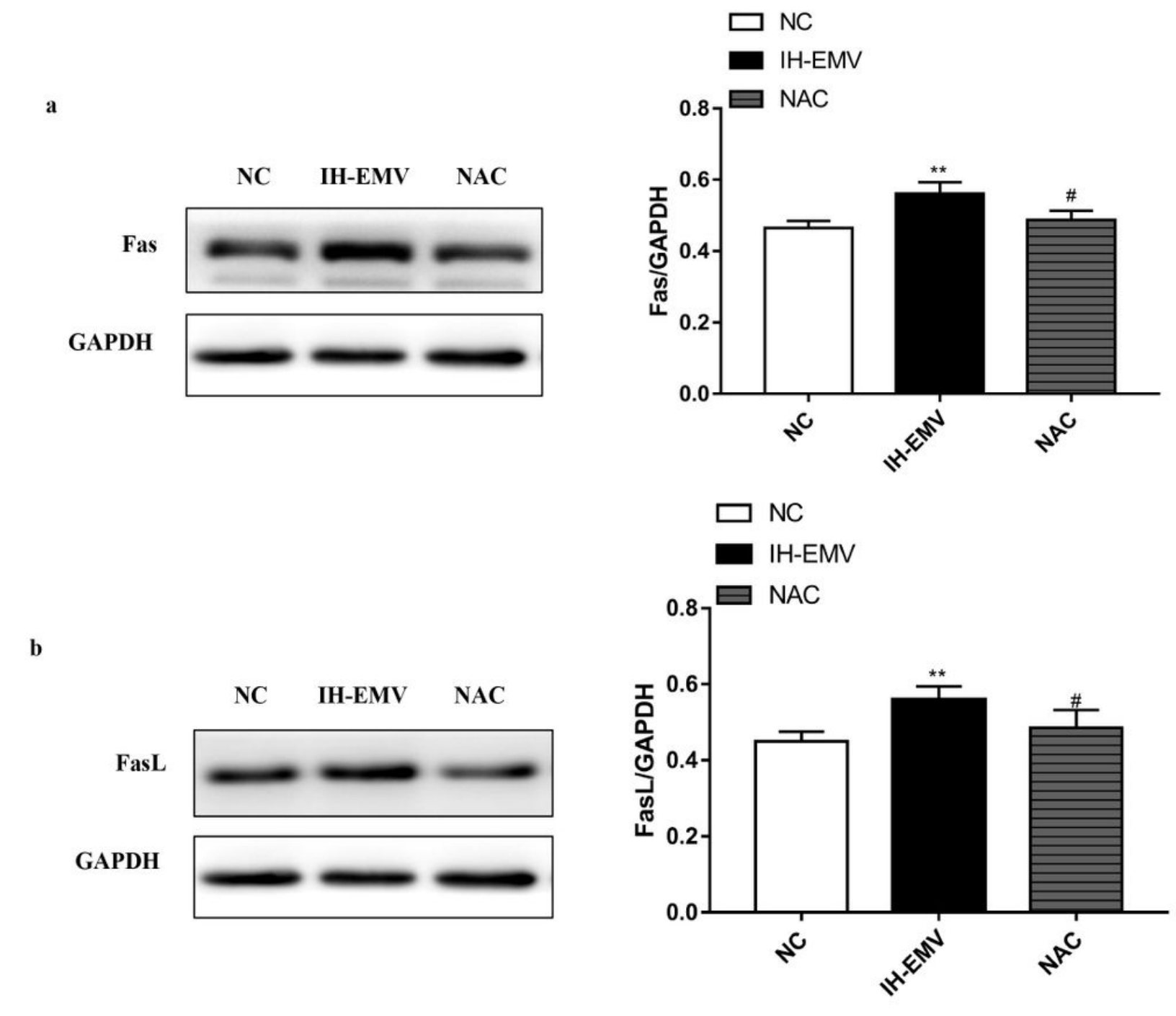

Figure 5

The up-regulation of Fas / FasL expression in neurocytes was related to the ROS IH-EMV carried. we added antioxidants NAC to the cell culture medium of IH group. (a) The expression of Fas in PC12 cells was assessed by Western blot in NC, IH-EMV and NAC group. (b) The expression of FasL in PC12 cells was assessed by Western blot in NC, IH-EMV and NAC group. ${ }^{\#} p<0.05$ vs NC, ${ }^{\# \#} p<0.01$ vs NC, ${ }^{*} p<0.05$ vs N-EMVs, ${ }^{* *} p<0.01$ vs N-EMVs. 
a

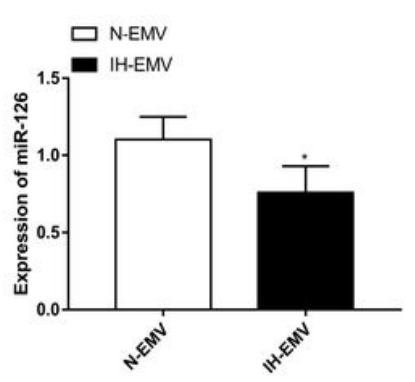

b

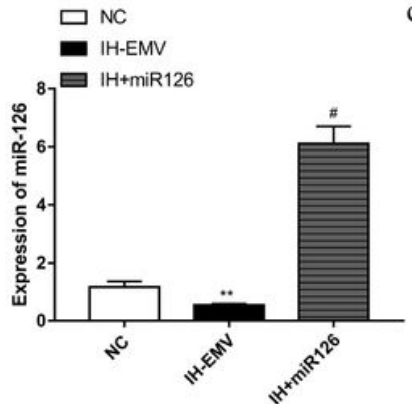

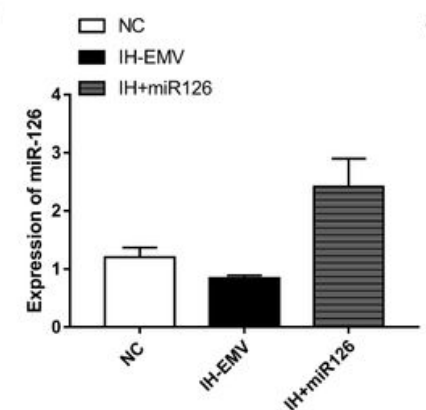

d $\square \mathrm{NC}$

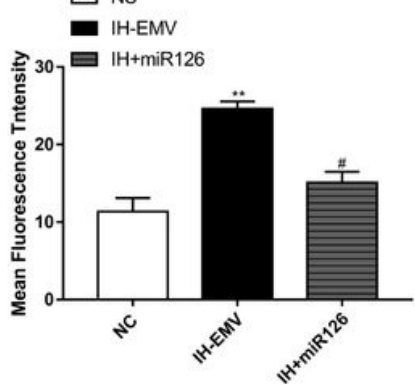

$\mathrm{e}$
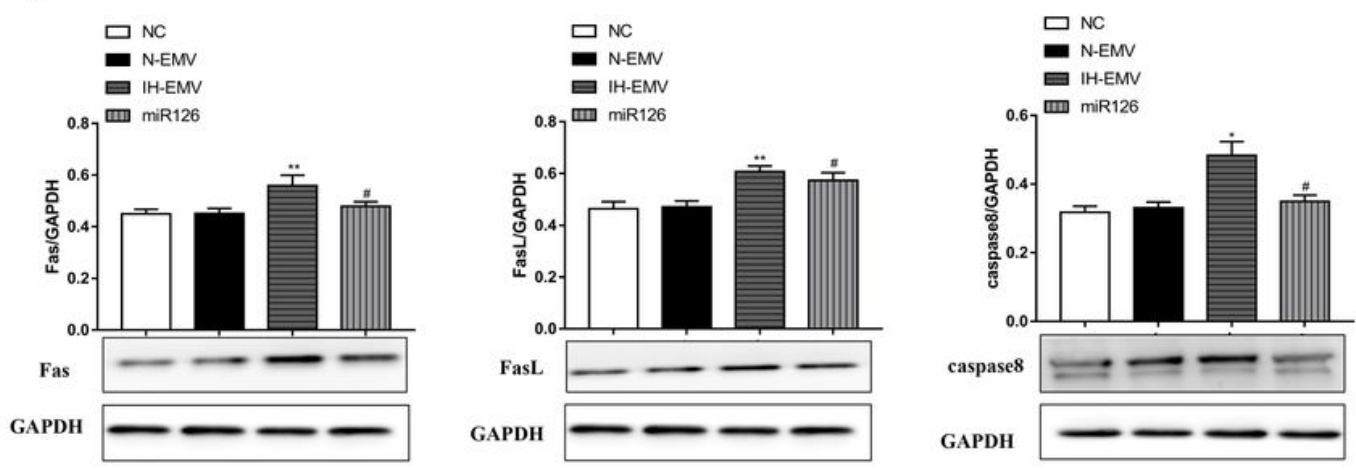

Figure 6

IH-EMV regulated the ROS-Fas/FasL-Caspase8 pathway through miR126. (a) The level of miR126 in NEMV and IH-EMV was assessed by RT-PCR. (b) The level of miR126 of B3 cells in NC, IH-EMV and $\mathrm{IH}+$ miR126 group. (c) The level of miR126 in N-EMV, IH-EMV and IH+miR126 group. (d) The level of ROS was assessed by Flow Cytometry in N-EMV, IH-EMV and IH+miR126 group. (d) The expression of Fas, FasL and caspase 8 in PC12 cells was assessed by Western blot in NC, N-EMV, IH-EMV and miR126 group. ${ }^{*} p<0.05$ vs NC, ${ }^{* \star} p<0.01$ vs NC, ${ }^{\#} p<0.05$ vs IH-EMV, ${ }^{\# \#} p<0.01$ vs IH-EMV. 


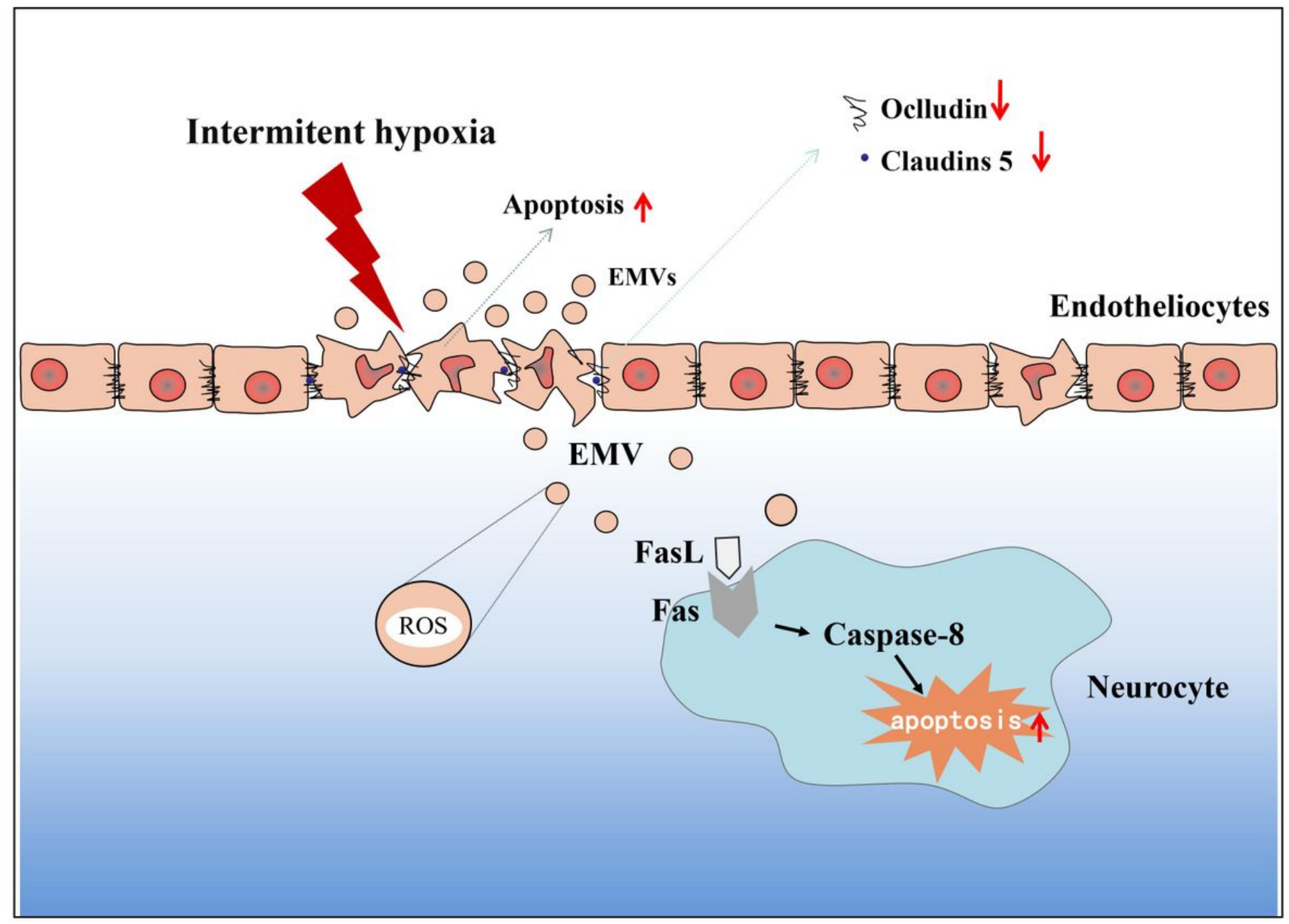

Figure 7

The cellular pathway 